\title{
Peut-on rester compétitif sur le marché sans faire appel aux capitaux extérieurs?
}

Le cas de la coopérative Leclerc

\section{How cooperatives can stay competitive in the market without external capital \\ The case of the Leclerc cooperative}

\section{Marie-Laure Baron}

Numéro 298, novembre 2005

URI : https://id.erudit.org/iderudit/1021842ar

DOI : https://doi.org/10.7202/1021842ar

Aller au sommaire du numéro

Éditeur(s)

Institut de l'économie sociale (IES)

ISSN

1626-1682 (imprimé)

2261-2599 (numérique)

Découvrir la revue

Citer cet article

Baron, M.-L. (2005). Peut-on rester compétitif sur le marché sans faire appel aux capitaux extérieurs ? Le cas de la coopérative Leclerc. Revue internationale de l'économie sociale, (298), 75-89. https://doi.org/10.7202/1021842ar

\section{Résumé de l'article}

Le développement coopératif est souvent traité sous l'angle des alliances, fusions, ouverture de capital... Comment une coopérative autocentrée, peu ouverte sur l'extérieur, peut-elle maintenir sa taille et sa compétitivité sur le marché ? La fidélisation des membres constitue un enjeu de taille que se propose d'analyser cet article, à travers le cas de la coopérative Leclerc, en s'appuyant sur la théorie des droits de propriété et sur la théorie de l'agence. La coopérative Leclerc a connu deux crises importantes dans son histoire; elles ont permis de mettre au point à la fois des incitations (système de parrainage) et des restrictions (limitation des possibilités de transfert) au droit de propriété, dans le but de fidéliser les adhérents et de les renouveler pour assurer un développement sur le long terme. La triple qualité de l'adhérent, apporteur de capital, utilisateur des services et décideur (obligation de consacrer un « tiers temps » à la gestion de la coopérative), facilite la conjonction des intérêts et limite les coûts d'agence. Si ces mécanismes ont prouvé leur efficacité, l'auteur montre qu'une extension de la taille de la coopérative paraît cependant compromise. 


\section{PEUT-ON RESTER COMPÉTITIF SUR LE MARCHÉ SANS FAIRE APPEL AUX CAPITAUX EXTÉRIEURS?}

\section{Le cas de la coopérative Leclerc}

(*) Maître de conférences en gestion, université du Havre, Cerene (Le Havre)-Crei (Paris-XIII). Mél. marie-laure.baron@univ-lehavre.fr.

\author{
par Marie-Laure Baron ${ }^{*}$
}

Le développement coopératif est souvent traité sous l'angle des alliances, fusions, ouverture de capital... Comment une coopérative autocentrée, peu ouverte sur l'extérieur, peut-elle maintenir sa taille et sa compétitivité sur le marché? La fidélisation des membres constitue un enjeu de taille que se propose d'analyser cet article, à travers le cas de la coopérative Leclerc, en s'appuyant sur la théorie des droits de propriété et sur la théorie de l'agence. La coopérative Leclerc a connu deux crises importantes dans son histoire; elles ont permis de mettre au point à la fois des incitations (système de parrainage) et des restrictions (limitation des possibilités de transfert) au droit de propriété, dans le but de fidéliser les adhérents et de les renouveler pour assurer un développement sur le long terme. La triple qualité de l'adhérent, apporteur de capital, utilisateur des services et décideur (obligation de consacrer un " tiers temps " à la gestion de la coopérative), facilite la conjonction des intérêts et limite les coûts d'agence. Si ces mécanismes ont prouvé leur efficacité, l'auteur montre qu'une extension de la taille de la coopérative paraît cependant compromise.
(1) Voir Guillouzo R., Perrot P., Ruffio P. (2002), par exemple, sur les alliances dans les coopératives agricoles.
D ans la concurrence que sont amenées à se livrer les firmes, le cas de la concurrence entre firmes coopératives et firmes intégrées pose différentes questions. On peut citer les difficultés de coordination de la firme coopérative, le sous-investissement chronique (Cook, 1995; Condon et Vitaliano, 1983), la lenteur du processus démocratique... Une autre particularité intéressante à étudier est celle relative à la variation de la taille de la coopérative, liée à sa structure contractuelle, et au principe de libre entrée et sortie des adhérents qui devrait la gouverner. La coopérative peut être instable (Vienney, 1994), tant sur le plan du capital (variable) que sur celui des volumes traités. Cette caractéristique de la coopérative la rend particulièrement fragile sur des marchés où les volumes produits ou affichés sont souvent un critère déterminant du prix ou du pouvoir de négociation.

Les coopératives sont encore présentes sur les marchés cependant, ce qui s'explique parfois par les transformations opérées. Les fusions, alliances, etc., sont l'un des axes stratégiques développés ${ }^{(1)}$. Une autre 
(2) Les SA se transforment en SAS depuis la circulaire Dutreil, qui limite à cinq le nombre de mandats d'administrateur possible pour une personne.

(3) Pour une vision complète du secteur, se référer à Moati (2003). évolution peut consister à transformer la coopérative en société de droit commun (sous les conditions de la loi de 1947, c'est-à-dire en cas de difficulté), ce qui permet, outre de faire appel aux capitaux extérieurs, de redessiner le mode de fonctionnement interne. Certaines coopératives existent encore, cependant, dans leur forme originelle, et parmi elles le mouvement Leclerc.

Le mouvement Leclerc est une coopérative de commerçants, réunissant des $S A^{(2)}$ (points de vente), ultimes destinataires des profits du réseau, au sein de coopératives d'achat régionales et d'une coopérative nationale (le Galec). C'est la firme " hybride " (Williamson, 1985) par excellence. Cette coopérative, créée dans les années 60 , a évolué au cours de près d'un demi-siècle, sans ouvrir son capital, sans faire appel à des fusions ou rachats, et en pratiquant les alliances à très faible dose (alliance avec Système U et Conad en Italie). Figure de la modernité, concurrençant le grand groupe intégré Carrefour sur le territoire français, elle a une authenticité coopérative qui justifie de s'intéresser à la manière dont sont coordonnées les activités et dont la taille de la firme est maîtrisée, dans un secteur où les effets d'échelle et la concentration son particulièrement importants ${ }^{(3)}$.

L'objet de cette contribution est de montrer comment une coopérative autocentrée, peu ouverte sur l'extérieur, parvient à stabiliser son périmètre et à rester compétitive sur le marché en dehors de tout appel à l'extérieur. L'approche développée ci-après, essentiellement empirique, s'appuie sur les apports de la théorie des contrats, et en particulier de la théorie des droits de propriété initiée par Demsetz (1967), puis appliquée au monde coopératif par Condon (1987). Après avoir explicité le problème de la gestion des entrées et sorties de la coopérative et montré comment la théorie des droits de propriété peut rendre compte de ce problème, la troisième partie de l'exposé est consacrée à la répartition des droits de propriété et des décisions chez Leclerc, pour mettre en évidence la cohérence et les limites d'un système performant et peu coûteux.

\section{Problématique de la libre entrée et sortie de la coopérative}

(4) La transformation de la coopérative est réglementée: elle ne peut se faire que lorsque la survie de l'entreprise ou les nécessités de son développement (lorsque celui-ci ne peut plus intervenir dans le cadre coopératif) l'exigent. Dans ce cadre, une coopérative peut alors être transformée en une société de droit commun après autorisation de l'autorité administrative (loi de 1947).
Pour Jacques Defourny (1995), là où la coopérative, comme forme d'organisation spécifique, ne répond plus aux besoins particuliers de ses adhérents, elle disparaît pour prendre éventuellement une autre forme (société anonyme...) ${ }^{(4)}$. Selon lui, les conditions d'existence de la coopérative sont, d'une part, qu'elle obéisse à une nécessité ( $c f$. sa vitalité dans les pays en voie de développement) et, d'autre part, qu'il existe une identité collective en son sein. Lorsque l'une de ces deux conditions est absente, logiquement la coopérative disparait, et c'est ce que l'on peut observer parfois dans la transformation des organisations mutuelles ou coopératives: n'ayant plus d'identité collective 
ou n'étant plus nécessaires à leurs adhérents, elles se transforment peu à peu.

L'interprète de la " nécessité de la coopérative " n'est autre que l'adhérent qui mesure l'utilité de la coopérative à l'aune de ses intérêts propres. Dans différents secteurs, la coopérative réunit différents entrepreneurs qui ont des intérêts convergents au moment de l'adhésion, mais qui peuvent, plus tard, souhaiter organiser leurs relations au marché différemment. Ainsi, lorsque les adhérents ont bénéficié du savoir-faire de la coopérative et ont grandi avec elle et qu'ils souhaitent réorienter leur activité, se pose la question de la sortie de la coopérative. La sortie peut être souhaitée pour d'autres raisons, comme le départ à la retraite d'un adhérent après vingt, trente ou quarante ans d'activité.

Ces sorties ne sont pas nécessairement compensées par des entrées en nombre équivalent ou en taille équivalente, auquel cas le capital de la coopérative, variable en fonction du nombre d'adhérents, peut se réduire de façon importante. En effet, les sorties ont tendance à se faire de manière groupée, soit qu'une génération remplace l'autre ou bien que la coopérative connaisse une crise d'identité (désaccords internes sur la politique adoptée), ou bien encore que le marché sur lequel la coopérative est positionnée soit lui-même en crise et conduise les adhérents à se retirer du marché. Dans les coopératives d'agriculteurs, par exemple, en fonction d'incitations gouvernementales ou d'opportunités de marché, l'exploitant peut renoncer à exploiter ou changer de type de plantation, auquel cas son adhésion à la coopérative de pommes quand il va faire autre chose n'a plus de sens.

Ces départs simultanés peuvent mettre la coopérative en grande difficulté et accélérer sa disparition. La coopérative se caractérise en effet par la « double qualité » des associés, qui sont à la fois apporteurs de capital et utilisateurs de la coopérative comme clients, fournisseurs ou salariés. La diminution du nombre d'associés a donc un impact direct sur l'activité de la coopérative (volume d'affaires) et sur son capital.

La stabilisation de la taille de la coopérative tient ainsi à une bonne gestion du " parc » d'adhérents à la coopérative. Suivant l'évolution du nombre d'adhérents, on peut se trouver selon les cas dans l'une ou l'autre des configurations présentées en page suivante. Dans la configuration 1, idéale, le nombre d'adhérents se développe, puis se stabilise durablement: schématiquement, le nombre de nouvelles entrées égale le nombre de sorties, la coopérative reste attractive pour de nouveaux entrants. La configuration 2 présente le cas d'une coopérative qui se développe rapidement, puis voit le nombre de ses adhérents s'effondrer rapidement. Les adhérents disparaissent et il n'y a pas de nouveaux entrants. Ce peut être le résultat d'une forte crise d'identité, de conflits importants entre les adhérents. Dans la configuration 3, on a une coopérative qui se développe, se stabilise, puis dont le nombre d'adhérents diminue progressivement, par exemple à la suite d'un phénomène générationnel: le solde des entrants et sortants est négatif et la coopérative disparaît peu à peu, ce qui pénalise les derniers adhérents. 


\section{Evolution d'une coopérative autocentrée en fonction du nombre d'adhérents}

Configuration 1

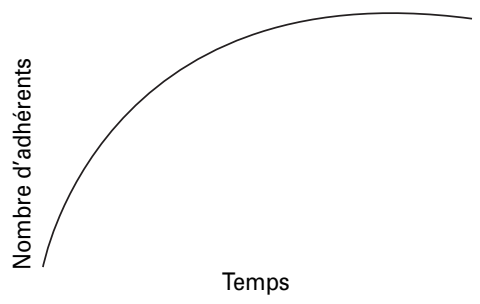

Configuration 3

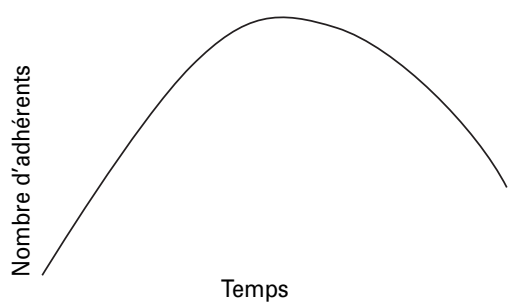

Configuration 2

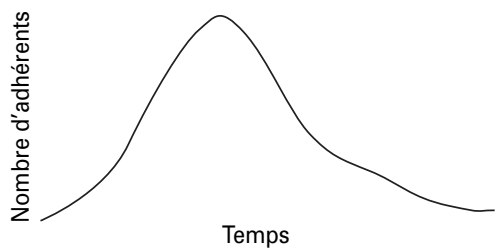

Configuration 4

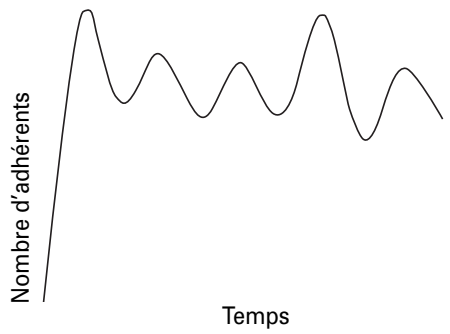

Enfin, la configuration 4 est celle qui nous intéresse. Elle montre le développement d'une coopérative avec progression du nombre d'adhérents, puis une série de crises qui vont faire varier ce nombre. Il peut s'agir de crises d'identité de la coopérative, de difficultés liées aux débouchés sur le marché ou au prix, qui entraînent le départ des adhérents. Ce qui est intéressant dans ce cas, cependant, c'est la capacité de la coopérative à réduire les effets des crises successives, c'est-à-dire à conserver une taille "à peu près " stable dans le temps. Autrement dit, malgré des crises où se manifestent des départs en série, la coopérative parvient à limiter les effets des départs, à recruter de nouveaux adhérents et à retrouver éventuellement un rythme de croisière.

Il est possible, en s'appuyant sur le modèle Leclerc, de montrer comment une coopérative peut gérer les crises et stabiliser sa taille. Mais cela suppose de comprendre les mécanismes organisationnels sous-jacents à la coopérative.

\section{Structure coopérative et contrat d'adhésion}

La coopérative se différencie de la firme intégrée classique. Elle existe par la seule volonté personnelle des adhérents d'y adhérer et d'y participer 
et par sa capacité, forte de ces adhésions, à rester sur le marché, à justifier son existence. La volonté des adhérents est formalisée dans le contrat d'adhésion. La coopérative constitue donc, au sens de la théorie de l'agence, un nœud de contrats.

Les difficultés liées à la gestion contractuelle des activités économiques ont été mises en évidence dans la théorie des contrats depuis quelques années déjà. Le risque principal est celui d'une appropriation de la valeur commune, générée dans l'exercice du contrat, par l'une des parties au contrat (Klein, Crawford et Alchian, 1978). L'incertitude est une composante essentielle de la problématique, particulièrement dans les contrats de long terme comme peut l'être un contrat d'adhésion à la coopérative. Les agents ayant une rationalité limitée (Simon, 1982), les contrats vont être conclus compte tenu de la situation du moment et de ce qui est prévisible. Le contrat d'adhésion est donc nécessairement incomplet. Il va pourtant être conclu pour une durée longue, au cours de laquelle apparaitront nécessairement des conflits d'intérêt entre les adhérents et la coopérative. L'incomplétude du contrat, jointe au conflit d'intérêts, crée la possibilité de départ d'un adhérent détenteur d'un actif spécifique (Williamson, 1975).

L'organisation qui gère ses activités économiques se trouve ainsi devant un double défi: celui d'organiser l'activité commune pour maximiser la valeur générée, d'une part, et celui d'éviter que l'une des parties ne s'approprie une trop grosse part de cette valeur, d'autre part. Or, dans le cas de la coopérative, le départ d'un ou plusieurs adhérents réduit la valeur générée et peut conduire à une appropriation de la rente par un petit nombre.

L'une des solutions préconisées par la théorie, pour limiter les risques liés à une telle appropriation, est d'intégrer les actifs spécifiques, c'est-à-dire les actifs qui ont une grande utilité pour la firme (Klein, Crawford et Alchian, 1978; Williamson, 1975). Les actifs des adhérents sont très spécifiques pour la coopérative, puisqu'ils sont sa raison d'exister. La coopérative dépend de ces actifs pour organiser sa relation au marché. Pourtant, par hypothèse ici, il n'est pas question d'intégrer ces actifs. Il faut donc organiser les activités de manière efficace, en limitant les risques, sans intégrer.

La théorie des droits de propriété et la théorie de l'agence sont sans doute plus adaptées pour traiter de la coopérative (voir par exemple K. Zeuli, 2004), parce qu'elles mettent en évidence la spécificité de la firme coopérative par rapport à la firme intégrée. La théorie des droits de propriété, initiée par Demsetz (1967), suggère que si les droits de propriété de chacun des participants à la firme sont correctement attribués, alors la firme peut être efficace. La bonne organisation, ici l'organisation stable, est alors celle qui, en fonction des droits de propriété concédés, incite les différents agents à se comporter conformément à l'intérêt du principal, le propriétaire.

La théorie de l'agence s'inscrit dans cette tradition, en étendant ce principe à l'ensemble des contrats. Elle définit une relation d'agence comme "un contrat par lequel une ou plusieurs personnes (le principal) engage une autre personne (l'agent) pour exécuter en son nom une tâche quelconque qui implique 
une délégation d'un certain pouvoir de décision à l'agent». En raison des divergences d'intérêts et de l'asymétrie d'information entre les deux parties, une telle relation engendre des coûts d'agence engagés par le principal pour obtenir le comportement adéquat de la part de l'agent: les dépenses de surveillance et d'incitation, les coûts d'assurance, le coût d'opportunité. Le problème est alors de déterminer l'organisation qui minimise les coûts d'agence.

La réduction des coûts d'agence dans l'organisation suppose une répartition des droits de propriété et des droits sur les décisions qui permette d'aligner au mieux les intérêts des uns et des autres. En particulier, l'attribution des droits de propriété aux créanciers résiduels, c'est-à-dire ceux qui supportent les risques, revêt une importance particulière. Dans la firme intégrée, trois catégories d'agents sont identifiées: les créanciers résiduels (associés, actionnaires), le management, qui prend les décisions, et la direction, qui contrôle ces décisions. Les coûts d'agence sont ceux rendus nécessaires pour rallier les intérêts de tous à ceux des propriétaires.

Si l'un des problèmes fondamentaux de la firme coopérative est de réguler sa taille, la structure des droits de propriété doit inciter les adhérents à contribuer à la performance de la firme et à rester dans son périmètre. La définition des droits de propriété doit permettre de fidéliser les adhérents et de les renouveler pour se situer ou bien dans la configuration 1 ou bien dans la configuration 4. Mais dans cette coopérative en particulier, il n'y a pas trois catégories d'agents comme dans la SA classique, typique des sociétés modernes: les trois agents sont quasiment confondus en un. On pourrait parler de la triple qualité de l'associé: apporteur de capital, utilisateur des services et décideur ou manager [pas de délégation au sens de Berle et Means (1932) au management].

\section{Définition des droits de propriété chez Leclerc}

Les coopératives de producteurs ou de commerçants sont organisées de telle sorte que les profits ne sont pas véhiculés vers le haut de l'organisation (les actionnaires ou associés), mais vers le bas, vers ceux qui participent à la coopérative. Il s'agit de coopératives de moyens qui ont pour objet de faciliter l'activité de leurs membres.

Dans le cas de Leclerc, le créancier résiduel (celui qui assume le risque), et le bénéficiaire ultime des profits, est le propriétaire du point de vente, adhérent à la coopérative. En adhérant à la coopérative, il transfere une partie de son autonomie en se soumettant à l'autorité du « collectif » coopérative; cependant, cette concession va de pair avec l'obtention d'avantages suffisants pour justifier ce transfert. La valeur globale générée collectivement dépend de la capacité de l'ensemble des adhérents à fonctionner de concert, et donc à générer de la valeur, et de la capacité de la coopérative à limiter les risques d'appropriation de la rente. 


\section{Présentation de la coopérative Leclerc}

- Taille: 480 adhérents, 73000 salariés.

- Enseigne $n^{\circ} 1$ en France en termes de parts de marché, deuxième groupe de distribution derrière Carrefour (qui recouvre plusieurs enseignes).

- Adhésion à la charte Leclerc comprenant cinq points:

- le respect de la politique de prix bas;

- la participation des salariés aux résultats de l'entreprise ( $25 \%$ des bénéfices réalisés sont distribués en fin d'année au niveau de chaque magasin);

- la contribution active à la vie du mouvement;

- la sécurité collective;

- le parrainage (obligation pour chaque adhérent de parrainer un nouvel adhérent: cautionner financièrement, aider...).

- Fonctionnement démocratique: " un homme, une voix ", avec un effort constant du groupement pour limiter les pouvoirs locaux. Ainsi, avec 480 adhérents, le groupement affiche environ 500 points de vente.

- Agrandissement par cooptation des nouveaux adhérents par le système du parrainage.

- Organisation de toutes les instances sous forme coopérative (centrale nationale, centrale régionale...), pour assurer le partage des bénéfices en fonction de la contribution de chacun.

(5) Dans le cas d'Intermarché, on retrouve également des crises de ce type, avec par exemple, récemment, le départ d'adhérents (et donc de points de vente) suite à la crise d'identité du mouvement au moment du départ du fondateur et de l'arrivée du nouveau dirigeant. Un grand nombre de coopératives connaissent ces crises, particulièrement au moment des changements d'orientation de la politique. (6) Les magasins de Bouliac, Cannes, Paris (groupe Abihssira) et Marseille quittent le mouvement Leclerc au profit des concurrents Auchan ou Promodès, celui de Neuville-Maisons (1995) au profit d'Intermarché.
Une partie du dispositif mis en place chez Leclerc est issue de l'évolution du groupement à travers deux crises ${ }^{(5)}$ notables dont la coopérative garde la mémoire:

- La crise de 1969, qui a donné lieu à la scission d'avec Intermarché. Celle-ci prend naissance lors d'une crise d'identité du mouvement Leclerc, à un moment où il atteint une certaine taille. Les adhérents discutent alors des modalités de gestion de la coopérative, et le conflit se solde par le départ d'environ soixante-dix adhérents.

- La crise des années 80-90(6), où les points de vente Leclerc sont la cible de groupes intégrés concurrents qui tentent de racheter individuellement les points de vente leur convenant le mieux. Les adhérents de la coopérative sont alors incités à céder leur point de vente au plus offrant des concurrents, au détriment des autres coopérateurs qui voient leur pouvoir sur le marché s'effriter.

Les dispositifs mis en place, notamment dans la restriction des droits de propriété, tiennent compte de ces expériences. Néanmoins, une incitation ancienne à rester dans la coopérative est liée aux profits que les adhérents pourront en tirer.

\section{Droits de propriété: incitations}

Les adhérents se partagent le surplus de la coopérative, qui est redistribué en fonction de la contribution de chacun à son fonctionnement (pourcentage du CA). Par ailleurs, chaque adhérent, dirigeant de son point de vente, 
(7) Le développement de la coopérative Intermarché, par exemple, beaucoup moins sélectif, peut être analysé comme entraînant des conflits internes sur les droits de propriété des différents adhérents. peut se verser un salaire. Les adhérents du mouvement Leclerc sont également propriétaires depuis peu de la marque Leclerc, qui leur a été cédée par le fondateur. A ce titre, ils sont collectivement maîtres de son devenir.

Mais l'incitation principale offerte par la coopérative est celle de pouvoir devenir adhérent, propriétaire de son point de vente, sans patrimoine de départ et de finir sa carrière en réalisant une forte plus-value personnelle. En effet, typiquement, l'adhérent Leclerc est un ancien directeur de point de vente Leclerc qui a été parrainé par un adhérent en particulier et par plusieurs autres pour prendre un point de vente. Par le biais de ce parrainage qui cautionne son démarrage auprès des banques, l'adhérent va pouvoir accéder à la propriété d'un point de vente à partir d'un minimum de ressources. A la fin de son parcours, il pourra revendre ce point de vente en réalisant une plus-value.

Le parrainage constitue donc une institution particulière qui remplit une quadruple mission:

- celle de sélectionner les futurs adhérents en fonction de leurs compétences professionnelles et de leur adhésion aux valeurs du groupement (procédure d'agrément). Ce contrôle des entrées facilite également l'attribution de droits de propriété lisibles pour les adhérents ${ }^{(7)}$;

- celle de surveiller les nouveaux adhérents par le biais du parrain principal, qui a une obligation d'accompagnement;

- celle d'assurer l'extension du réseau et le renouvellement des adhérents;

- celle d'assurer le financement des nouveaux adhérents.

C'est sur ce mécanisme que repose en grande partie la stabilité du groupement.

En cas de difficulté de montage du parrainage, le mouvement Leclerc a également mis en place un système de "portage ", qui permet de reprendre les points de vente et de les «porter " durant une période transitoire. Ainsi les statuts des SCA régionales évoluent-ils pour intégrer une nouvelle mission, celle de reprendre des points de vente. L'article 2 (objet de la société), alinéa $f$, précise que la société peut « acheter des fonds de commerce dont [...] la location-gérance sera concédée dans un délai de deux mois à un associé et qui devront être rétrocédés dans un délai maximum de sept ans". Parallèlement, les statuts du Galec, centrale d'achat du groupe, prévoient la possibilité pour un gérant (et pas seulement pour un propriétaire de point de vente) d'être associé, membre du directoire ou du conseil de surveillance. Ces mécanismes solidaires vont permettre de gérer le décalage temporel entre entrées et sorties de la coopérative.

Si les incitations sont relativement anciennes, le parrainage a été mis en place à la suite de la première crise du groupement, lors de la scission des adhérents Intermarché (1969). Ce mécanisme de surveillance va être complété, à la suite de la seconde crise, d'autres éléments institutionnels visant à apporter des restrictions aux droits de propriété.

\section{Les restrictions aux droits de propriété des adhérents}

La deuxième crise du mouvement Leclerc apparaît nettement comme une série de comportements opportunistes en l'absence de clause 
(8) Quatorze décisions du tribunal de Nanterre confirment qu'exiger vingt-cinq ans de présence n'est pas excessif, que les pénalités de départ ne sont pas abusives et, enfin, que le refus d'adhérer au " pacte de préférence " provoque I'exclusion par anticipation de I'adhérent (affaire Fiscel). Tribunal de Nanterre, 1998.

(9) Mais on a vu ci-dessus que différents mécanismes étaient envisagés.

(10) Soit 750000 euros environ pour un point de vente réalisant un $C A$ de 150000000 euros, soit environ $10 \%$ du prix de vente d'un point de vente. contractuelle précise. Elle constitue un hold-up de la part de quelques-uns, vendant au plus offrant, au détriment des adhérents restant dans la coopérative. Pourtant, du point de vue de la coopérative, c'est grâce à l'enseigne gratuite et au support de la coopérative que ces différents points de vente ont pu atteindre une telle valeur. Cette crise peut mettre à mal l'esprit coopératif et la coopérative elle-même, qui perd du terrain par rapport à ses concurrents et donc du pouvoir de négociation. Dans différentes affaires, la coopérative a pu obtenir gain de cause devant les tribunaux ${ }^{(8)}$.

La réaction du groupement est cependant d'apporter des restrictions aux droits de propriété en limitant les possibilités de transfert et la liberté d'entrée et sortie de la coopérative. Sont introduits une durée de contrat minimale, une obligation de préavis en cas de départ, une pénalité en cas de départ anticipé et un droit de préemption de la coopérative. La crise des années 80-90 entraîne donc un renforcement des statuts de la coopérative. Au niveau national est introduite en 1991 une clause selon laquelle «les adhérents au 25 juin 1990 sengagent à demeurer membres du Galec (coopérative d'achat du mouvement) pour une durée égale au moins à vingt-cing ans, durée qui sera au moins égale à trente ans pour les nouveaux adhérents". Concernant le droit de préemption du mouvement Leclerc sur les points de vente introduit en 1994 : tout adhérent partant doit proposer son point de vente en priorité à la coopérative, qui, en cas de rachat, trouvera un adhérent pour le reprendre. Cette clause permet de conserver un front de vente stable, à condition de: a) trouver un nouvel adhérent répondant aux critères du groupement; b) trouver les financements nécessaires à la reprise $^{(9)}$.

Les pénalités prévues en cas de non-respect des règles sont relativement inopérantes: la menace d'exclusion reviendrait à se passer d'adhérents précieux (des points de vente, donc des débouchés) pour rester compétitif. Les départs avant le délai de vingt-cinq ou trente ans sont par ailleurs difficiles à éviter quand l'évolution du mouvement conduit à intégrer des adhérents de 40 ans et plus. La pénalité prévue au contrat du Galec n'est par ailleurs pas extrêmement dissuasive: $0,5 \% \mathrm{du} C A$ du point de vente ${ }^{(10)} \mathrm{et}$ renoncement aux ristournes non encore versées.

Ainsi, l'évolution du groupement entrâne une restriction des droits de propriété des adhérents, avec la création d'un marché interne du point de vente Leclerc. L'adhérent est ainsi limité dans son pouvoir de transfert.

Par ailleurs, d'autres règles viennent limiter les coûts d'agence. Ainsi, celle qui limite chaque adhérent à deux points de vente maximum réduit le pouvoir de négociation de l'adhérent individuel et permet une meilleure gestion des points de vente.

\section{Organisation et prise de décisions}

Chomel et Vienney (1995) attribuent la disparition de Codec, coopérative de distribution, à l'absence d'implication des adhérents et à la prise des 
décisions (de pouvoir) par les salariés. Il semble donc important d'impliquer les adhérents dans les processus de décision.

Le choix adopté par Leclerc est de faire participer les adhérents au fonctionnement de la coopérative. Le refus de participation aux instances du mouvement, significatif d'un comportement opportuniste, autorise le groupement à exclure l'adhérent de plein droit (statuts de la coopérative d'achat nationale). Cette pratique parfois décriée, appelée le tiers temps, donne l'obligation à chaque adhérent de consacrer deux jours par semaine au groupement. Chaque adhérent, outre la direction de son (ses) point(s) de vente, s'implique dans les achats nationaux, dans la gestion ou la direction des centrales régionales ainsi que d'autres établissements (bases d'importation, par exemple) et dans les " groupes de travail » qui travaillent sur les projets de la coopérative. Chaque adhérent a une tâche au sein de la coopérative, et notamment celle de s'occuper des achats sur un marché donné pour l'ensemble des points de vente adhérents. L'adhérent ayant une bonne connaissance de la problématique point de vente est incité à effectuer les achats dans les meilleures conditions pour la défense de l'intérêt des points de vente. Par ailleurs, il dépend de l'ensemble des autres adhérents pour les achats des autres produits dans son point de vente. Chacun est ainsi client et fournisseur de ressources, chacun est client des uns et fournisseur des autres. Le système construit facilite la conjonction d'intérêts entre la coopérative et ses adhérents et crée un système de surveillance par les pairs du type de celui souligné par Mintzberg (1982) dans la bureaucratie professionnelle.

\section{Droits de propriété dans la coopérative Leclerc}

Droits de propriété

Propriété

de la marque Leclerc

Profit courant de la SA

Salaire

Plus-value sur la revente du point de vente

Possibilité de développer le site "magasin " avec adjonction de nouveaux services
Décisions

Restrictions au droit de propriété

Achats nationaux, internationaux, locaux qui ont un effet sur la performance des points de vente Gestion de son point de vente

Choix de son filleul

\section{Droits de propriété:}

- obligation de vendre à prix bas

- risque d'exclusion

- limitation du nombre de points de vente par adhérent

- limitation sur la revente du point de vente

- caution du nouvel arrivant

\section{Décisions :}

- contrôle par les pairs 
La distribution des droits de propriété et des décisions donne corps à la firme, qui peut bénéficier d'un périmètre identifiable, conserver sa taille et rester sur le marché. Le système crée une incitation à rester dans la coopérative, avec la possibilité pour chaque adhérent d'augmenter son patrimoine par le développement de son point de vente, mais aussi par la pression des pairs qui ont permis ce succès. L'adhérent est incité à investir, puisqu'il pourra bénéficier des fruits de ces investissements lors de la revente. La stratégie du groupe est d'ailleurs de développer les sites de chacun des adhérents, par un développement horizontal de l'activité (parapharmacie, espace culturel, parfumerie, magasin de sport, cinéma...).

Il existe cependant des limitations à ces droits de propriété, puisque chaque adhérent est limité dans le nombre de sites et dans les possibilités de transfert. Les décisions prises par les adhérents, segmentées par créneaux de marché spécifiques, sont cohérentes avec les objectifs des adhérents et de la coopérative (améliorer la performance en réduisant le coût d'achat); leur répartition crée un système d'autosurveillance. Les décisions de parrainage permettent de recruter et de sélectionner les meilleurs adhérents pour l'avenir.

\section{Commentaires sur le cas particulier de Leclerc: portée et résultats}

(11) La contrainte d'horizon provient du fait que, lorsque l'adhérent investit, il n'est pas certain de pouvoir bénéficier des gains de cet investissement compte tenu du délai de retour sur investissement et de son départ probable.
Un premier commentaire peut être fait, à ce stade, pour nuancer l'analyse proposée. D'abord, la coopérative Leclerc bénéficie de quelques avantages particuliers:

- Le fait que le dirigeant de la coopérative soit le fils du fondateur, ce qui lui donne une légitimité et limite les conflits internes sur les décisions stratégiques. Dans le cas de Leclerc, d'une certaine façon, au lieu d'avoir comme dans la firme intégrée à coordonner des dirigeants, un management et des créanciers résiduels, il n'y a presque qu'un seul corps, celui des adhérents, managers et dirigeants à la fois, ce qui limite les coûts d'agence.

- Le fait que le marché de la grande distribution se porte assez bien et que les adhérents puissent faire évoluer leur activité vers des créneaux plus porteurs (parapharmacie, culture, sport) sans remettre en question leur appartenance à la coopérative, ce qui réduit la pression sur les « créanciers résiduels " que sont les adhérents (perte de valeur de leur investissement, contrainte d'horizon ${ }^{(11)}$ telle que soulignée par Porter et Scully, 1987). La création d'un " portefeuille " de services sur un site limite en effet les risques supportés par l'adhérent (contrainte de "portefeuille ", Cook et Iliopoulos, 1998). L'activité étant porteuse, le retour sur investissement rapide, les investissements dans la coopérative sont facilités.

- Le fait que la politique du distributeur soit axée depuis l'origine sur les prix bas, ce qui facilite la coordination des activités, puisque chacun, dans la coopérative, intègre cet objectif qui permet, comme le proposent Fama et Jensen (1983), "de rester sur le marché parce que la demande est satisfaite au meilleur prix ». Cette obligation interne au groupement de vendre moins 
cher limite les profits des adhérents, mais leur donne une accroche particulière sur le marché et permet d'authentifier une communication immuable depuis de nombreuses années.

Le caractère conjoncturel des crises, et notamment de la seconde, avec l'augmentation du prix des points de vente ne modifie en rien la problématique du cas. En effet, quel que soit le contexte de la " crise ", celle-ci constitue un moment de fragilité pour la coopérative. Par définition, la crise est inattendue par certains aspects et peut s'aggraver en s'appuyant sur l'incapacité de l'institution (du contrat) à réguler la relation avec les adhérents. C'est dans ces moments que les mécanismes organisationnels à l'œuvre dans la coopérative pourront révéler leur efficacité.

Lorganisation adoptée par le mouvement Leclerc paraît donc vertueuse du point de vue des adhérents collectivement, si l'on tient compte de l'hypothèse de départ qui est celle de la non-intégration.

Les coûts de surveillance sont minimes, puisque celle-ci est assurée par le fonctionnement quotidien du système. Les échanges fréquents entre les adhérents à différents niveaux (région, local, différentes instances) favorisent la communication interne et limitent les chances d'occurrence d'un conflit d'intérêts. Le parrainage, par exemple, crée de la valeur en même temps qu'il limite les risques d'appropriation.

Les risques d'opportunisme sont réduits par les restrictions sur les droits de propriété. Le coût de l'opportunisme effectif peut cependant être évalué par les coûts des procès intentés par la coopérative aux adhérents qui ont quitté le groupement dans des conditions un peu particulières. Certains de ces procès se soldent d'ailleurs par des dédommagements significatifs pour Leclerc.

Les incitations sont coûteuses pourtant, puisque ce sont elles surtout qui vont permettre la fidélisation des adhérents. D'une certaine manière, en effet, il est possible de considérer que le coût de fidélisation des adhérents correspond au profit que chacun pourra tirer de sa participation à la coopérative. Ce profit correspond au profit dégagé par le point de vente une fois les prêts bancaires remboursés, ainsi quà la plus-value encaissée lors de la revente, l'adhérent n'ayant qu'un faible apport à l'origine. Chaque année, une trentaine de points de vente au moins changent de main, dont la cession est financée par le système du parrainage. Ce sont donc chaque année autant de ressources qui sont exclues du système de production de la coopérative et sont perdues pour des développements futurs. Les parrainages utilisés pour financer les successions ne sont pas utilisés pour développer le groupement. Fait symptomatique d'ailleurs, là où il fallait seulement un ou deux parrains autrefois, il en faut parfois aujourd'hui jusqu'à huit pour assurer le transfert. Le système risque donc l'épuisement si le nombre de sorties augmente trop. Entre 0,5 et $1 \%$ du chiffre d'affaires annuel du groupement, dans un secteur où le résultat net avoisine 2-3\% du chiffre d'affaires, s'échapperait ainsi chaque année. Le système est vertueux pour les adhérents, mais pas nécessairement pour la coopérative, qui doit nécessairement réduire son développement. Peut-être d'ailleurs y aurait-il un 
lien à faire entre le faible développement de la firme à l'étranger et l'importance des successions à assurer en France.

\section{Conclusions et enseignements}

La présentation faite ici de la coopérative est naturellement " stylisée » en raison du choix effectué de faire appel à la théorie des droits de propriété qui focalise l'attention sur les incitations. Elle ne rend pas bien compte, par exemple, de la forte identité collective qui existe du fait des échanges nombreux entre les adhérents et de leur identification comme des indépendants par rapport à la firme intégrée. La confiance est un autre ciment du groupement, qui agit « en creux » et qui est peu abordé ici.

La gestion de la taille de la coopérative se fait donc ici par l'organisation du remplacement des adhérents partants, la limitation à la sortie et la sélection à l'entrée, qui permet de bénéficier de managers compétents et respectant les principes internes. Les crises entraînent un renforcement du contrat à la fois en longueur (durée) et en épaisseur (obligations) au profit de la coopérative. La stabilisation de la firme passe par une restriction des droits de propriété, notamment en ce qui concerne le transfert des actifs spécifiques, et par un allongement de la durée du contrat.

Le cas montre que la répartition des droits de propriété et des prises de décision ainsi que les mécanismes de coordination adoptés sont de nature à réduire les coûts d'agence. La coopérative Leclerc présente une distribution des droits de propriété et des décisions qui devrait permettre une convergence d'intérêts entre les différents membres, mais aussi inciter ces derniers à œuvrer au développement de la coopérative, chacun à sa manière. En même temps, il existe différents modes de surveillance, mais qui sont peu coûteux pour la firme. Les incitations à s'impliquer et à participer dans la coopérative sont très fortes, ainsi que les incitations à investir dans le point de vente dont la reprise est " garantie " par le mouvement Leclerc. La création d'un marché interne du point de vente (et des parts de coopératives associées) correspond aux préconisations de Cook et Iliopoulos (1998) pour inciter les partenaires à investir. La coopérative est rendue nécessaire à ses adhérents en ce qu'elle leur permet de s'enrichir. Ainsi, Leclerc organise la double, voire la triple qualité de l'adhérent.

Les limites au système sont évidentes: limite à la taille en raison d'une grande mobilisation de ressources dans l'entretien du réseau (beaucoup moins coûteux à entretenir dans le cas d'un réseau en franchise, par exemple), épuisement du système de renouvellement si le système de parrainage est étouffé par un trop grand nombre de partants successifs, chute collective en cas de retournement du marché et épuisement radical des ressources (type configuration 3, voir le schéma page 78). Une autre limite peu développée ici vient du fait que la promesse d'enrichissement offerte aux adhérents peut prendre le pas sur l'identité collective et l'esprit coopératif. 
Si tel était le cas, le risque serait de voir les départs se multiplier dès que les adhérents estimeraient avoir réalisé un gain suffisant, par exemple à l'issue des remboursements d'emprunts. Les règles de la coopérative, avec le système de pénalités, limitent le risque, mais seraient probablement inopérantes si un nombre suffisant d'adhérents souhaitaient partir. La contrainte sur la taille de la coopérative est alors le contraire de la contrainte d'horizon évoquée précédemment: les profits autorisés sont tellement élevés que les adhérents n'ont pas d'incitation à rester au-delà d'un certain montant (ils deviennent rentiers). La coopérative se retrouve dans la même situation qu'au moment de la crise des années 90.

On remarquera également l'évolution vers une intégration croissante de la coopérative pour garantir sa taille par le développement d'un contrat de long terme avec les adhérents, caractéristique de la " quasi-intégration ». A lui seul, ce dernier serait sans doute inopérant pour retenir les adhérents. Mais cet allongement de la durée est symptomatique de la réduction de l'instabilité de la firme hybride. La procédure d'agrément à l'entrée et le contrat de long terme créent une limitation à l'entrée et à la sortie de la coopérative. Par ailleurs, l'évolution se produit après une crise qui apparaît ici comme une modalité d'apprentissage.

Enfin, on observe que, si la coopérative se transforme peu en apparence, elle se transforme de l'intérieur de manière importante tout de même. Une extension de ce modèle reste cependant limitée par rapport à d'autres coopératives (outre les limites soulevées ci-dessus), puisque dans le modèle présenté ici, tous les adhérents peuvent participer à la coopérative. Ce n'est pas toujours possible, les besoins en management ou peut-être les besoins financiers n'étant pas aussi importants dans d'autres coopératives. 


\section{Bibliographie}

Berle et Means (1932), The modern corporation and private property, Commerce Clearing House, NY.

Chomel et Vienney (1995), "Evolution des principes et des règles des organisations coopératives en France (1945-1992) ", in Coopératives, marchés, principes coopératifs, De Boeck Université, "Ouvertures économiques", pp. 117-154.

Condon (1987), "The methodology and requirements of a theory of modern cooperative enterprise ", in J. SS Royer ed., Cooperative theory: new approaches, US Department of Agriculture, Agricultural Cooperative Service, ACS service report $\mathrm{n}^{0} 18$, Washnigton DC, pp. 1-32.

Condon et Vitaliano (1983), "Agency problems, residual claims, and cooperative enterprise ", cooperative theory working paper $n^{\circ} 4$, Department of Agricultural Economics, Virginia Polytechnical Institute and State University. Cook M. (1995), "The future of us agricultural cooperatives: a neo-institutional approach ", American Journal of agricultural economics, 77 (December), 1153-1159.

Cook et Iliopoulos (1998), " Property rights constraints in collective action: the case of US agriculture producer groups ", $2^{\text {nd }}$ conference of the International Society of new institutional economics, Paris 1998.

Defourny J. (1995), introduction de Pratiques coopératives et mutations sociales, L'Harmattan, "Logiques sociales ".

Demsetz (1967), « Toward a theory of pro- perty rights ", American Economic Review, 57 (2), pp. 347-59.

Fama et Jensen (1983), «Separation of ownership and control ", Journal of law and economics, 26, 301-325

Guillouzo, Perrot, Ruffio (2002), «Stratégies d'alliances et configurations des groupes agricoles: les apports d'une analyse en termes de portefeuille d'alliances ", Recma, n 285 , pp. 75-92.

Klein, Crawford et Alchian (1978), « Vertical integration, appropriable rents and competitive contracting process ", Journal of law and economics, 21, pp. 297-326.

Mintzberg H. (1982), Structure et dynamique des organisations, pp. 310-335.

Porter et Scully (1987), « Economic efficiency in cooperatives ", The Journal of law and economics, 30 (October), pp. 489-512.

Simon H. (1982), "A behavioural model of rational choice ", in Models of bounded rationality and behavioral economics and business organization, Cambridge, MIT press.

Vienney C. (1994), L'économie sociale, La Découverte, "Repères ", Paris.

Williamson (1975), Markets and hierarchies, analysis and antitrust implications, New York Free Press.

Williamson (1985), The economic institutions of capitalism, New York Free Press.

Zeuli K. (2004), "The evolution of the cooperative model ", Cooperatives and local development, theory and applications for the $21^{1 t}$ century, edited by Christopher D. Merret and Norman Walzer, 2004, pp. 52-69. 